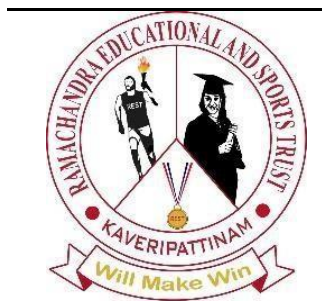

Data Analytics and Artificial Intelligence

Vol: 1(1), 2021

REST Publisher

ISBN: 978-81-948459-4-2

Website: http://restpublisher.com/book-series/data-analytics-and-artificialintelligence

\title{
A Review on Differential Evolution Optimization Techniques
}

\author{
C. Venkateswaran, M. Ramachandran, M. Amudha, T. Vennila, M. Manjula \\ REST Labs, Kveripattinam, Krishnagiri, TamilNadu, India. \\ Email: venky.professor@gmail.com
}

\begin{abstract}
In evolutionary calculation, differential evolution (DE) is a method of developing a problem in an evolutionary process. Such algorithms do not make some or all assumptions about the basic optimization problem and can quickly explore very large design gaps. Differential Evolution (DE) is a complete search algorith mbased on population, which is based on a candidate's evolutionary process. Improves the problem by repeatedly upgrading the solution. Such algorithms give little or no speculation about the basic optimization problem and will quickly explore the larger design gaps; both genetic algorithms and differential evolution are examples of evolutionary calculations. Genetic mechanis ms are very clo se to the metaphor of genetic reproduction. ... Different evolution is in the same style, but correspondence is not precise. DE is the processing of the population of individuals represented by the dimensional vectors of real numbers Is an evolutionary mechanism. In every repetition, in every parent, a mutation is a different mutation driver Created by The mutant then communicates with the parents and gives the offspring a different evolution (DE) a competitive form of evolutionary mechanis ms. It relies heavily on modifying solutions, using measured differences of approximately selected individuals from the population, to develop new solutions. Differential reproductive success refers to the statistical analysis that compares successful reproduction rates between groups in a given generation of a species - in other words, how well each individual group is able to leave offspring, with examples of both genetic mechanisms and different evolution. Evolutionary calculation. Genetic mechanis ms are very close to the metaphor of genetic reproduction. ... Different evolution is in the same style, but correspondence is not precise. DE is the processing of the population of individuals represented by the dimensional vectors of real numbers Is an evolutionary mechanis $\mathrm{m}$. In each iteration, for each parent, one with a different mutation driver the mutant is created. The mutant then goes to the parents and gets an offspring
\end{abstract}

\section{Introduction}

Phonetic species concept (morphological species concept): a collection of organis ms that is identical and different from othe $\mathrm{r}$ groups. Biogenetic species concept: a very small monophyletic group that may differ by shared derived (synapomorphic) characteristics. What are the different species? A Species are a group of organisms that share a genetic heritage that can reproduce and produce fertile offspring. Breed different species are separated from each other by barriers. Is one of the mos $t$ specific classifications used by scientists to describe animals? Sympatric specialization is the evolution of a new species from a surviving ancestral species, while the two continue to live in the same geographical area. In evolutionary biology and biogeography, sympathy and empathy are terms that refer to organisms, and their boundaries occur together in certain places. An invasive species is an organism that is neither native nor native to a particular area. Invasive species can cause great economic and environmental damage to the new area. What is differential evolution? Different evolution occurs when the population of an animal or plant is divided into two groups by geographical barrier (e.g., aquatic or migration to a new area), with each group developing different characteristics under their respective selections. Stresses and natural selection involve unrelated organisms that develop similar traits over time, while differential evolution involves species with common ancestors in that particular environment. Non-native: a species that originated somewhere other than its current location and was introduced to the area where it now lives (also known as exotic species). 'Co-evolution' occurs when independent species acquire the same characteristics when they are formed together at the same time in the same space. In parallelevolution, the ancestors of the respective lineages were somewhat similar in terms of that trait. Hoops in biology and phylogenetic are when a trait is acquired or lost in separate lineages during evolution. Woodblocks can arise from both the same selection stresses and the effects of genetic slip on species adaptation. Different Boundaries represent areas where the plates expand. Change the borders where the plates slide over each other. Introduced race is a non-native species that is integrated into the native environment by human or other means in one way or another. on the other hand, is an invasive species introduced organism, which is harmful to the local environ ment. Bird, bat, and stroller wings have similar structures, but their foreleg s are identical and share ancestral status despite performing different functions. The opposite of aggregation is different evolution, in which related species develop different traits. This is an important difference. The true meaning of "native speaker" is often discussed, but it is generally accepted that a child learns the language as a child by listening to his parents speak. speakers of other languages learn the language as older children or as adults. 


\section{Parame ter Adaptation}

Since its beginning, DE has been as often as possible utilized for worldwide improvement issues, including a genuine capacity and restricted powers over dynamic factors. Starting around 2010, most single-reason DE sorts have been adjusted to boundary variation plans and numerous transformation and alternate route systems Have been changed. Use. Thusly, the limit between the strategies depicted in this segment and the techniques talked about in the past segment may not be so huge. Nonetheless, in this segment, notwithstanding essential determination and boundary variation strategies, DEs utilize altogether more complete techniques to improve their inquiry moves. We are exploring the techniques. We survey DE calculations distributed in pre-positioning diaries and gatherings that cover part of the developmental estimation. We will examine such DE varieties under the accompanying seven headings, albeit such gathering is generally easy, because a proposition can utilize a blend of strategies of various pioneers. These pioneers are DE new startup procedures, DE new or further developed transformation methods, new or further developed alternate route strategies, region and group based enhancements, segment variety and socioeconomics with individual probabilities of DE, DE. DE with area and parent determination framework [1]. The one-page algorithm is created by combining the test vector development the strategy and control parameter adaptation schemes mentioned above in a typical DE framework. In the SaDE algorithm, test vector generating techniques and both related parameter values are gradually self-altered by learning from previous experiences creating reliable solutions. Because of this, the most appropriate strategy and its parameter structure are modified to suit different stages of the search process Can be determined. The detailed tests described in Section IV test the reliability of the page in dealing with issues with unique characteristics such as similarity and diversity. The algorithm of the page is illustrated [2]. From the above, it can be observed that various conflicting conclusions have been drawn regarding the manu al parameter tuning of the DE. is not sufficiently justified. Various techniques have been developed to avoid correcting parameters by trial and error process. F can reduce the criterion. The control parameters are F and CR. FADE modifies control parameters F and CR based on obscure logic controllers, and its FADE performs better than conventional DE on performance values and high dimensional is sues associated with inputs. DE (ADE) parameter adaptation and diversification approach population It was proposed to control diversity, which was later expanded to create an adaptive Pareto DE algorith $\mathrm{m}$ for diversity improvement. Simultaneously with other parameters Update is sues arise by encrypting the crossover rater for each person. The F criterion was developed using the Gaussian distribution for each individual [3]. Adaptation of test Vector generation strategies and control parameters can be described as follows. In itially, the in itial population had a balanced distribution in PG Using; we create approximately three control lists for each target person. These lists are a mutation scaling factor F list, crossover ratio CR list and test vector build strategy list Are listed as belonging to the st rategic package referred to in Section 3.1, and the size of these lis ts is LP. Therefore, each target vector has an LP to generate an offs pring Contains a set of parameter values for generations. The proposed DE algorith m was started with the opposite $1 p^{1 / 41}$. During the evolutionary process, the parameters F and CR and the test vector development strategy S were set. for each target person and then used to create a new test vector. If the derivatives are better than its target, the relevant and $S$ Successful F list, the successful CR list and the successful strategy list, respectively wSLi. The generation counter is increased and the above evolutionary process is repeated [4]. To increase the strength of JADE, we propose an improved version of JADE that uses a different parameter adaptation mechanism based on the success -history-based Adaptive DE of successful parameter systems. In the shed, the average values of SCR and SF for each generation are stored in the MCR, MF historical memory. Unlike JADE, which guides parameter adaptation using a single pair, SHADE maintains a variety of parameters that guide control parameter adaptation as the search progresses. Therefore, even if SCR and SF have bad values for some generations, the parameters stored in memory from the previous generation cannot be directly negatively affected. It should be much stronger than JADE [5]. The second important area is the indirect ML rating of the configuration parameters, which provides the HMM para meter rating with some variations. It is abbreviated at the top left of the road map. Once the configuration of these two key areas is established, the MAP rating approaches can be applied to the configuration parameter as shown in the left outer corner of the block below the road map. The hybrid ML/ MAP rating can be done by combining the direct and indirect rating as shown in the right branch of the left block under the road map. Finally, the systematic development of integrated MAP evaluation of transformation and HMM parameters serves as an integrated framework for incorporating direct and indirect clas sification parameter evaluation / adaptation into the design of adaptive decision rules for automated speech recognition. It is worth noting that the combination of the above two parameters shown below and the on-line joint rating classifier provides a natural extension for changing parameters. Many technical integration paradigms can also be developed [6]. After defining the potential, it was decided to combine the input variables c1 and c2 to create different ambiguous settings For dynamic adjustment. Seven vague systems, based on combinations of possible inputs were, but decided to consider only systems with higher inputs (previously only one) We considered ambiguous systems with input), and finally there were three ambiguous systems. They are defined below. The first vague system is repeated and diversified entries, the second has repetitive and erroneous entries, and the third a mbiguity is repeated as duplicate and error entries, as shown in the design rules. For each ambiguous system, the PSO algorithm was explored at initial repetitions and finally it was decided that it should be used. Considering other variables such as diversity, the probe should be used when the diversity is low, i.e. when the particles are close, and exploitation should be used when the diversity is high. The rules for each ambiguous system are shown in ambiguous systems 1, 2 and 3, respectively. To compare the proposed method without parameter adaptation with PSO, we considered the benchmark mathematical functions, of which a total of 27 are defined, and we need to find the parameters. give the universal minimum size of each function in each. Figure 12 shows a model of the functions used [7]. 


\section{Global Nume rical Optimization}

As mentioned in Section III, the characteristics of the test vector generating techniques and the control para meters of the DE are examined in detail, and some obtained in the last decade Prior knowledge. Such prior knowledge can be used to design the most effective and robust DE types. In most DE types, including adaptive and self-adaptive DE types, the control parameter is a control for the system We also noticed that only the vector and test vector generating technique are used. As a result, the search capability of these algorithms may be lower. Based on the above considerations, CoDE We propose an innovative method called, whose primary idea is to approximately combine multiple test vector generation techniques with multip le control para meter systems for each generation to generate new test vectors [8]. This section briefly reviews some of the recently developed DE types related to the method proposed in this paper. Section III-A and Section III-B are mutation strategies and crossovers, respectively Showing the latest improvements in operators. Section III-C revie ws some innovative methods in integrating multiple mutation strategies. Section III-D examines recent advances in adaptive DE algorithms in strategy adaptation. Section III-E Control parameter for recent DEs Introducing in adaptation. Section III-F identifies the latest DE methods, proposing alternatives to parental selection during mutations and shortcuts. Section III-G shows the latest progress in population structure and activities. This Along the way, the most appropriate strategy can be determined in an adaptive manner. In addition to parameter adaptation and strategic adaptation, parental choice is a potential adaptation. Proximity-based mutation operators propose an alternative to parental uniform random selection during mutation, but parental selection that can create successful solutions has not been used [9]. This technique Allows the user to expand and contract the search space, i.e. polyhedron, Without special control variable settings. Unfortunately, the Nelder\& Mead method is basically a local reduction method, although our experiments introduce the concept of annealing, but not enough power for global reduction tasks. However, DE buys the idea of using information from vector persons to change the search location from Nelder\& Meat. DE's self-regulatory scheme is different from the approximately selected population vectors to disturb the existing vector Takes the vectors. Every population vectors are disturbed. This important idea contradicts the method used by traditional ESs to determine vector disturbances with predetermined probability distribution functions. Last but not least, the good integration properties required for a good little algorithm are mandatory. Although there are many approaches to theoretically describing the integrated properties of the global mitigation system, only extensive testing can show them under different conditions. that the mitigation system can deliver on its promises. The DE scores in this case are very good, which will be explained in detail [10]. For homogeneous and separable functions, rapid integration was provided (only $2 \%$ higher in f04 function). For multimedia and separable problems, all four types provided similar behavior in the f08 process, but, again, were faster in the other process, with the same performance problem obtained in f03 (identical and inseparable). Finally, for multimodal and inseparable problems, one-fifth to one-third provided faster integration, thereby actually showing much faster integration into the problem. Problem to be solved. However, if the problem is multimodal and inseparable, it will be a bit faster [11]. It is generally believed that most population-based optimization methods (except perhaps some hybrid global-local methods) encourage individuals (here, specifications of test vectors) to model different Search location zones. Early stages of the search. In the latter stages it is important to adjust the movements of the test solutions neatly so that they are optimal. Can explore the interior of a small space that is universally suspected. To achieve this goal, the value of the measuring factor is linearly linear (predetermined). Subtract from the maximum (predetermined) value: the maximum and minimum values of the measuring factor are the current iteration number and MAXIT is the maximum number. Repetitions. May be allowed. The location of the best vector node in the population under this scheme can thus be explained follows [12]. Before solving the optimization problem; We may not have any information about the global minimum location. The chromosomes of the early population are likely to be identical throughout the solution site Scattering is desirable so that the algorithm explores the entire solution base evenly. An orthogonal sequence refers to a small nu mber of combinations equal to the spacing of all possible combinations We notice that. Therefore, orthogonal design is a viable method for generating a good initial population. A factor that has factors for each chromosome We define. These factors are continuous, but orthogonal design applies only to individual factors. To deal with th is problem, we measure each factor as a finite value. Specifically, we measure the domain [13]. In multimodal operations, ugly and rotated ugly Easily solved by all Adaptive DE types with 100\% success rate. For Grie wank and Rastrigin, before the cycle, SaDE, ADE and jade had higher success rates as shown in Table IV. Had. After cycling (and), Side and Jade only found global optimization in a few streams, making SaDE more successful than JDE Gives the rate. No algorithm is too difficult to find a global optimal. $\mathrm{SaDE}$ can find optimal solutions to the last two joint problems, with s maller fixed deviations and greater success With ratios, it exhibits better performance and stability than the other three algorithms. From the results of the 30-D journals shown in Table V, we can observe the algorithms. did not succeed in achieving the same ranking in the 10-D journals and further improving. However, for average and constant deviations, SaDE gets smaller values on the ground, and jDE gets second smaller values to get smaller values [14]. Global genetic selection: Genetic selection is usually done among the parent population based on the fitness values of the individuals; a person with excellent fitness value is more likely to survive in the mating pool. In the current standard GA, local selection the result is a global selection of renewed populations. There are several selection methods, including roulette wheel selection, random global selection and ranking selection, among others the current study uses what is known as competitive selection, which is commonly found in the EP. Accepted, which allows you to control this selection process. Exam pressure (probability ratio of choice between qualified and less qualified individuals). In general, the co mpetitive selection is an individual to the mating pool each time (Chromosome). It first selects an approximate number of individuals from the parent population, then sorts them and sends the best ones to the mating pool. The above process is repeated until the mating pool is full [15]. 


\section{Gene tic Algorithms (Gas)}

The following performance measurements were Used in comparative analysis: 1) accuracy of final clustering results; 2) merging speed; And 3) strength (i.e., the ability to produce approximately the s a me results in repeated runs). Five real in the test set selected for this paper There are life datasets. Finally, an interesting application of the proposed algorithm to il lustrate the automation of some well-known grayscale images is illustrated. The rest of this paper is organized as follows. Section II Defines the clustering problem in formal language and provides a brief overview of previous work in the field of evolutionary partition clustering. Section III outlines the proposed ACDE algorithm. Section IV, five used for tests Describes real data sets, simulation technique, algorith ms used for comparis on and their parameter structure. The results of five real-life datasets and their application in image pixel classification are presented in Section V. The results are given in Section [16]. Compared to other EAs, DE is very simple and straightforward to implement. The main part of the algorith $m$ is the first four to encrypt in any programming language Takes five lines. Because simplicity of code is important for practitioners in other fields They may not be an expert in programming and solve their domain-specific problems They are looking for an algorith $m$ that can be easily implemented and tuned. Although PSO encryption is very high simple, it is efficient DE and its variants is far superior to the PSO variants with a variety of issues. Due to its simplicity, DE exhibits better performance compared to GX such as PCX, MA-S2, ALEP, CPSO-H and many more. And many more. Although some strong EAs such as CMAES can beat DE in CEC competition, the overall performance of DE in terms of accuracy, accumulation speed and strength is still attractive for a variety of real applications, even in objective operations. The proble ms of global optimization are so great that an approximate solution cannot be found in a reasonable time [17]. Restoration is the process by which chromosomes selected from a parent population are reconnected to form members of a hereditary population. The idea is to simulate the combination of genetic material that can occur when organisms reproduce. Because the choice for rearrangement is in favor of greater fitness, the balance of probabilities (with confidence) produces more compatible chromosomes. Redesign involves two main components, the gene operator shortcut and the mutation. Genetic operators do not make decisions in their behavior. Each occurs with a certain probability and the exact effect of the shortcut or mutation has not been determined. Crossover operator refers to the mixing of genetic material from two parent chromosomes selected to create one or two baby chromosomes. After selecting two parent chromosomes for rearrangement, a random number of gaps created with uniform probability and compared with a predetermined "cross ratio". If the rando m nu mber crossover rate is exceeded, no crossover occurs and one or both parents move on to the next level or reconnect without change [18]. We are now in a position to describe how to apply the power of genetic algorith ms to the type of learning method depicted in Figure 2. The main idea is to define the location of structures that are allowed to be explored by GAs. This Each point in space represents the "genetic material" of a task subsystem, and when injected into this system, its working capacity is now well defined and measurable. Learning strategy, of tested structures Utilizes GAs to maintain population and create new structures with better performance expectations. To maximize sophistication and complexity when considering the types of structura 1 changes that can be created in a task subcommittee There are different approaches. The simplest and most straightforward approach is for GAs to change the set of parameters that control the behavior of a redesigned, configured performance program [19]. the most frequently cited example of the mechanism of Neo-Darwinism is the piston petularia moth. Prior to the Industrial Revolution in England, the most common form of this moth (known as typhoid) was pale with small black spots - Against predators, the light of the lichen-covered tree trunks was well hidden against the background. With an increase in pollution, the tree trunks gradually darken, and the altered dark of carbon aria The form spread further. Natural selection in the predatory form of the bird is considered to be due to loss of fitness of the common form and consequent spread of carbon aria. Recently, pollution control as an adjunct to the law, the typhoid form has become widespread again. Following this type of process, a GA is gradually adapted to their 'environment' - in this case, externally imposed 'fitness' measurements, allowing people to reproduce with variations of possible solutions to mortal or upgrade problems [20]. The algorith ms were tested with population sizes of 200 and 500. All test runs were monitored for 500 generations. Michalewicz presented an empirical inquiry into the hierarchical performance of evolutionary projects and the loss of performance He points out that generalization develops, while Solomon analyzes the advantages of commonly used small mutation rates and focuses on the performance loss of conventional GAs. Integration in his dissertation Theoretical analysis provided by the system shows that the most widely used experimental functions have $\mathrm{n}$ independent parameters, and many GAs use complex scales when developing such functions. Have. The genetic and evolutionary algorithm describes several experimental functions implemented for use with the toolbox. These functions are derived from the literature on genetic algorith ms and the evolutionary techniques of global optimization [21]. Upgrade issues are insufficient and difficult to use. The most realistic method is to find several Pareto-optimal solutions at once so that the decision makers can select the most suitable solution for the current situation. Knowledge of many Pareto-optimal solutions can be useful for later use, especially in the current situation Change and new solution must be implemented. Because genetic algorithms handle the population of points rather than one point, many in one run can capture prototype solutions. In the following section, we describe GAS and current and previous studies process for solving multisector optimization problems [22].

\section{Artificial Intelligence}

Unlike other evolutionary methods, differential evolution (DE) does not use some probability distribution function to introduce variations in populations. Conversely, between randomly selected vectors (individuals) as a source of random variations for the third vector (individual) referred to as the target vector DE uses differences within. Test by adding weight difference vectors in the target vector Solutions are being developed. This process is referred to as the target vector change 
mutation operator. It is used as a restructuring or shortcut to create offspring that will only be accepted if the parents' fitness improves. The basic DE algorithm describes three evolutionary operators in detail below: mutation, shortcut, and selection [23]. Synthetic neural networks with the structures shown in Figure 2 have been proposed and trained using other methods to classify the equilibrium-p proble $m$. The example training package was similar to the test package and contained $2 p$ vectors. The following values of the parameters are assumed: For each algorithm: the error back-spread algorithm (EBP) and the Levenbeg Marquardt algorithm (LM) are executed 10 times and the mean values of the results are given. Everything else The methods are considered to be the same result criteria as the DE-ANNT method described in Section III of this paper. A learning coefficient equal to U 0.2 is considered in the EBP algorithm. An initial investigation was conducted earlier the tests began [24]. Since then, the advancement of artificial intelligence has been mainly in the following ways. Programs are written to solve a type of problem that causes humans intellectual problems: playing chess or checkers, revealing mathe matical principles, translating one code expression into another, provid ing $\mathrm{n}$ rules, integrating expressions that include basic functions, and determining mass-spectrophotographic and other data. When I design these projects, more or less general intellectual algorithms are identified d sometimes motivational, sometimes mathematical analys is, sometimes experiments with human $n$ subjects. Experimental programs can sometimes lead to a better understanding of intelligent methods and the discovery of new ones [25]. In recent years, artificial intelligence technologies have evolved dramatically due to advances in computer processing capabilities and the accumulation of large amounts of data. However, current artificial intelligence Technologies are limited to specific areas of knowledge such as results, image recognition, speech recognition and speech response. That is, current AI means convolutional neural networks (CNN) or continuous neural networks (RNN) or deep residual learning. Is a special type of artificial intelligence that acts intelligently on an individ ual, such as having deep neural networks? (DNN) stands for Speech Recognition and Learning to Understand Speech (RL). All of these are carried out in every part of the human brain Is part of intellectual work; They are only an alternative and do n ot perform all the functions of the human brain. In other words, AI cannot cooperate with whole brain functions such as selfunderstanding, self-control, self-awareness and self-motivation. In particular, the limitations of the latest artificial intelligence technologies are as follows [26]. Our framework does not support warning perspectives that emphasize the catastrophic consequences of automation for labor. On the contrary, it exemplifies many counter-forces that Softening the impact of automation on labor. Most Importantly, as we argued in the previous subsection, a key part of the adjustment process in dealing with rapid automation is the new labor-intensive Is to create tasks. Creating new jobs is not just about making money from heaven. New that gains strength when automation intensifies The re are good reasons why market incentives deliberately lead to job creation There are. Moreover, the most limited automation technologies of our time like AI can create the site creating some new tasks and jobs [27]. Can be replaced by well-established physics methods, and new ones can be extracted by sensory methods. Knowledge Pure form is not enough to meet all the needs of the economy. Support for the mind should be in addition to support for the body. As a result, the system and Knowledge must be transferred within the institutions involved within the basic tests of society. Semantics of knowledge, manageable, stored, transmissible, must be reset and translated into an easy-to-use format. Attracting the person who created it. Capturing, storing and reusing all the knowledge processes of technology discovered in the techniques of AI intelligent solutions has become the biggest challenge over the past decades [28]. In all of the above methods, multiple objectives are combined to create a purpose using some knowledge of the problem to be solved. Single- The objective upgrade guarantees a barre tooptimal solution, but a single-point solution. Decision makers in real world situations often require different alternatives in decision making. Also, these methods may not work effectively if certain objectives are noisy or have uninterrupted variable space. Some of these methods are expensive because they require customized knowledge prior to vector optimization. The most profound drawback of these algorithms is their sensitivity to weights or demand levels [29].

\section{Particle Swarm Optimization (Pso)}

Some documents modify DE projects by mixing DE programs with other algorithm systems. Papers Lynn et al. And Su and Lee mixed integer / true representation Propose hybrid DE algorithms for classified problems. The particle in the handless DE is mixed with the swarm optimizer. And Zhang et al. Hybrids of DE with Ant Colony Optimizer Are proposed. Write the DE plan with the hybrid mutation rule. Paper Him and Hahn are a hybrid DE based on artificial immune systems Propose. Das et al. The proposed hybrid is with simulated annealing. Introduced the concept of super-fit adaptation and used to integrate various algorithm components. In addition to the Historical data is another source that can be used to improve integrated performance, with better solutions for the current population. An example is particle mass optimization (PSO), to guide the current population (mass) movement The best solutions in the history used. In this paper, we are more interested in the recently explored non-standard solutions than the best solutions previously explored, and from the current population. We see it as a promising direction to improve diversity. These solutions are also used to improve population diversity. In view of the above considerations, the preferred Introducing a new greed mutation mechanism called "DE / Current-to-Best" with external archive, which we use based on our parameter adaptive algorithm JADE. An earlier version of the JADE algorith m provided [30]. Box plots Based on 27 averages of comparative success rates calculated from 100 runs per task. Relative success rate for all types of DEs Values are significantly lower than CRS4. The average success rate of CRS4 is approximately $42 \%$, which is higher than the maximum value for all DE types. Of DE Reel-variations slightly increase the relative success of random localization compared to their standard counterparts, thereby increasing the reliability of the search. Improves and improves integration. The average relative success rate of RIL-variants is significantly smaller than the relative success rate of CRS4, which is suspected to be the reason for the slowdown. aggregation compared to CRS4 [31]. Here, we refer to population size, proble m size and The population size becomes N, D and NG respectively. First, initially, 
the time complex of ADDE is $\mathrm{O}(\mathrm{N} \times \mathrm{D})+\mathrm{O}(\mathrm{N})$, which is derived from the MASTER function, in ADDE In terms of individual evolution, ESE is implemented first, and its time co mplex is $\mathrm{O}(\mathrm{D})+\mathrm{O}(\mathrm{N})$, which is then derived., ADDE sends the whole population from MASTER to three slaves study develops simultaneously by improving the three populations, the exploitative population and the equilibrium population., And normal persons respectively [32].The proposed DECC-I and DECC-II are designed to include mechanisms that are highly effective for inseparable functions. First, both DECC-I and DECC-II are original one-dimensional Extend decay patterns. They improve the subgroup of all variables simultaneously rather than one variable. Also this will increase the chance of upgrading some interactive variables together. Also, became DECC-I It has additional weighing mechanis ms to collaborate on different groups of interactive variables, whi le DECC-II always modifies the optimal subgroup to increase the probability of grouping interactive variables together. Although DECC-I and DECC-II are not optimal for some current functions, they provide a starting point for further refinement in the field of high-dimensional optimization [33]. Inspired with the potential and potential of DE, many researchers are now working on its development, resulting in many variations of the algorith $\mathrm{m}$. A brief overview of these contemporary research efforts in this area Is provided. Fan and Lambinen proposed a new version of the DE that uses an additional mutation function called trichonometric mutation function (TMO). This modified DE algorithm is TDE The algorithm is called DE (TDE). In fact, TDE uses a probability mutation program, in which the new TMO and the original different mutation function are randomized. Used. Introducing an additional control parameter for random mutation, they showed that the TDE algorithm outperforms the classic DE algorithm for some definitions and real-world problems [34]. Tested uncontrolled problems include rosin rock activity, generalized rostrigrin, and generalized treebank activity, all of which have the same minimu $\mathrm{m}$ value of 30-D and zero. lists the average exercise values of activities such as FPSO (m) with different nu mbers of agents. For the parameters for these functions since there is little correlation between, the CR is set to small. DEPSO is be tter than two older PSO versions this shows that it works, especially for Rastrigrin and Griewank operations, which have unrelated parameters [35].

\section{Conclusion}

Since its origin, DE has been often utilized for worldwide improvement issues, including a genuine capacity and restricted powers over dynamic factors. Starting around 2010, most single-reason DE sorts have been adjusted to boundary transformation plans and different change and easy route techniques Have been altered. Use. In this manner, the limit between the We audit DE calculations Published in pre-positioning diaries and meetings covering a part of the developmental computation. About such DE varieties under the accompanying seven headings we will examine, in any case, that such gathering is generally easy on the grounds that a proposition can utilize a mix of methods from vario us pioneers. This Leaders DE with new startup strategies, DE with new or further developed transformation procedures, DE with new or further developed alternate route methods, region and group based enhancements, segment variety and populace direction with DE, DE individual probabilities. DE with area and parent choice framework. Area III-C surveys a few inventive techniques in coordinating different transformation methodologies. Area III-D analyzes the most recent advances in versatile DE calculations in technique transformation. Area III-E presents the most recent DEs in the control boundary variation. Area III-F distinguishes the most recent DE techniques, proposing options in contrast to parental choice during transformations and alternate routes. Segment III-G shows the most recent advancement in populace design and exercises. Thusly, the most proper methodology not entirely set in stone in a versatile way. Each happens with a specific likelihood and the specific impact of the alternate way or change has not entirely settled. Hybrid administrator alludes to the blending of hereditary material from two parent chro mosomes chose to shape a couple of youngster chromosomes. Subsequent to having two parent chromosomes The arbitrary number of holes chose for rev amp $[0,1]$ is created with a similar likelihood and contrasted and the foreordained "cross proportion". The reel-variations of the DE marginally increment the overall accomplishment of arbitrary confinement contrasted with their standard partners, according ly working on the unwavering quality of the pursuit and working on the mix. The normal relative achievement pace of reel variations is essentially lower than the overall achievement pace of CRS4, which is suspected to be because of their slow incorporation with CRS4 models: play ing chess or checkers, demonstrating numerical hypotheses, changing coding articulations, $\mathrm{n}$ articulations and synchronization articulations Determination of accumulates viable with spectrographic and different information. At the point when I plan these undertakings, pretty much broad scholarly calculations are recognized d here and there persuasive, at times numerical examination and in some cases explores different avenues regarding human $\mathrm{n}$ subjects.

\section{Reference}

1. Das, Swagatam, SankhaSubhraMullick, and Ponnuthurai N. Suganthan. "Recent advances in differential evolution-an updated survey." Swarm and evolutionary computation 27 (2016): 1-30.

2. Qin, A. Kai, Vicky Ling Huang, and Ponnuthurai N. Suganthan. "Differential evolution algorithm with strategy adaptation for global numerical optimization." IEEE transactions on Evolutionary Computation 13, no. 2 (2008): 398417.

3. Mallipeddi, Rammohan, Ponnuthurai N. Suganthan, Quan-Ke Pan, and Mehmet FatihTasgetiren. "Differential evolution algorithm with ensemble of parameters and mutation strategies." Applied soft computing 11, no. 2 (2011): 1679-1696.

4. Pan, Quan-Ke, Ponnuthurai N. Suganthan, Ling Wang, Liang Gao, and RammohanMallipeddi. "A differential evolution algorith m with self-adapting strategy and control parameters." Computers \& Operations Research 38, no. 1 (2011): 394408 . 
5. Tanabe, Ryoji, and Alex Fukunaga. "Success-history based parameter adaptation for differential evolution." In 2013 IEEE congress on evolutionary computation, pp. 71-78. IEEE, 2013.

6. Lee, Chin-Hui, and QiangHuo. "On adaptive decision rules and decision parameter adaptation for automatic speech recognition." Proceedings of the IEEE 88, no. 8 (2000): 1241-1269.

7. Melin, Patricia, Fru men Olivas, Oscar Castillo, Fevrier Valdez, Jos e Soria, and Mario Valdez. "Optimal design of fuzzy classification systems using PSO with dynamic parameter adaptation through fuzzy logic." Expert Systems with Applications 40, no. 8 (2013): 3196-3206.

8. Wang, Yong, Zixing Cai, and Qingfu Zhang. "Differential evolution with co mposite trial vector generation strategies and control parameters." IEEE transactions on evolutionary computation 15, no. 1 (2011): 55-66.

9. Guo, Shu-Mei, Chin-Chang Yang, Pang-Han Hsu, and Jason S-H. Tsai. "Improving differential evolution with a successful-parent-selecting framework." IEEE Transactions on Evolutionary Computation 19, no. 5 (2014): 717-730.

10. Xue, Feng, Arthur C. Sanderson, and Robert J. Graves. "Pareto-based multi-objective differential evolution." In The 2003 Congress on Evolutionary Computation, 2003. CEC'03., vol. 2, pp. 862-869. IEEE, 2003.

11. Mezura-Montes, Efrñn, Jesús Velázquez-Reyes, and Carlos A. CoelloCoello. "A comparative study of differential evolution variants for global optimization." In Proceedings of the 8th annual conference on Genetic and evolutionary computation, pp. 485-492. 2006.

12. Leung, Yiu-Wing, and Yuping Wang. "An orthogonal genetic algorithm with quantization for global numerical optimization." IEEE Transactions on Evolutionary computation 5, no. 1 (2001): 41-53.

13. Qin, A. Kai, Vicky Ling Huang, and Ponnuthurai N. Suganthan. "Differential evolution algorithm with strategy adaptation for global numerical optimization." IEEE transactions on Evolutionary Computation 13, no. 2 (2008): 398417.

14. Tu, Zhenguo, and Yong Lu. "A robust stochastic genetic algorithm (StGA) for global numerical optimization." IEEE Transactions on Evolutionary Computation 8, no. 5 (2004): 456-470.

15. Das, Swagatam, Ajith Abraham, and Amit Konar. "Automatic clustering using an improved differential evolution algorithm." IEEE Transactions on systems, man, and cybernetics-Part A: Systems and Humans 38, no. 1 (2007): 218237.

16. Das, Swagatam, and PonnuthuraiNagaratnamSuganthan. "Differential evolution: A survey of the state-of-the-art." IEEE transactions on evolutionary computation 15 , no. 1 (2010): 4-31.

17. McCall, John. "Genetic algorithms for modelling and optimization." Journal of computational and Applied Mathematics 184, no. 1 (2005): 205-222.

18. De Jong, Kenneth. "Learning with genetic algorithms: An overview." Machine learning 3, no. 2-3 (1988): 121-138.

19. Reeves, Colin, and Jonathan E. Rowe. Genetic algorithms: principles and perspectives: a guide to GA theory. Vol. 20. Springer Science \& Business Media, 2002.

20. Digalakis, Jason G., and Konstantinos G. Margaritis. "An experimental study of benchmarking functions for genetic algorithms." International Journal of Computer Mathematics 79, no. 4 (2002): 403-416.

21. Digalakis, Jason G., and Konstantinos G. Margaritis. "An experimental study of benchmarking functions for genetic algorithms." International Journal of Computer Mathematics 79, no. 4 (2002): 403-416.

22. Salman, Ayed, Andries P. Engelbrecht, and Mahamed GH Morin. "Empirical analysis of self-adaptive differential evolution." European Journal of operational research 183, no. 2 (2007): 785-804.

23. Slowik, Adam, and Michal Bialko. "Training of artificial neural networks using differential evolution algorithm." In 2008 conference on human systeminteractions, pp. 60-65. IEEE, 2008.

24. McCarthy, John, and Patrick J. Hayes. "Some philosophical problems from the standpoint of artificial intelligence." In Readings in artificial intelligence, pp.431-450. Morgan Kaufmann, 1981.

25. Lu, Huimin, Yujie Li, Min Chen, Hyoungseop Kim, and Seiichi Serikawa. "Brain intelligence: go beyond artificial intelligence." Mobile Networks and Applications 23, no. 2 (2018): 368-375.

26. Acemoglu, Daron, and PascualRestrepo. 8. Artificial Intelligence, Automation, and Work. University of Chicago Press, 2019.

27. Dean, Thomas, James Allen, and Yiannis Aloimonos. Artificial intelligence: theory and practice. Benjamin-Cummings Publishing Co., Inc., 1995.

28. Srinivas, Nidamarthi, and Kalyanmoy Deb. "Muiltiobjective optimization using no dominated sorting in genetic algorithms." Evolutionary computation 2, no. 3 (1994): 221-248.

29. Neri, Ferrante, and Ville Tirronen. "Recent advances in differential evolution: a survey and experimental analysis." Artificial intelligence review 33, no. 1 (2010): 61-106.

30. Zhang, Jingqiao, and Arthur C. Sanderson. "JADE: adaptive differential evolution with optional external archive." IEEE Transactions on evolutionary computation 13, no. 5 (2009): 945-958.

31. Tvrdík, Josef. "Adaptation in differential evolution: A numerical co mparison." Applied Soft Computing 9, no. 3 (2009): 1149-1155.

32. Zhan, Zhi-Hui, Zi-Jia Wang, Hu Jin, and Jun Zhang. "Adaptive distributed differential evolution." IEEE transactions on cybernetics 50, no. 11 (2019): 4633-4647.

33. Yang, Zhenyu, Ke Tang, and Xin Yao. "Differential evolution for high-dimensional function optimization." In 2007 IEEE congress on evolutionary computation, pp. 3523-3530. IEEE, 2007.

34. Noman, Nasimul, and Hitoshi Iba. "Accelerating differential evolution using an adaptive local search." IEEE Transactions on evolutionary Computation 12, no. 1 (2008): 107-125. 
35. Zhang, Wen-Jun, and Xiao-Feng Xie. "DEPSO: hybrid particle swarm with differential evolution operator." In SMC'03 Conference Proceedings. 2003 IEEE International Conference on Systems, Man and Cybernetics. Conference ThemeSystem Security and Assurance (Cat. No. 03CH37483), vol. 4, pp. 3816-3821. IEEE, 2003.

36. Gadde Mehar Chaitanya, M.P.Jenarthanan, C. Sathiyaraj, "A Review on Glass fibre Reinforced Composites with Different Matrix", REST Journal on Emerging trends in Modelling and Manufacturing, 7(1), (2021):18-24.

37. R. Kurinjimalar, S. Vimala, M. Silambarasan, S. Chinnasami. "A Review on Coir fibre Reinforced Composites with Different Matrix", REST Journal on Emerging trends in Modelling and Manufacturing, 7(2), (2021):25-32.

38. Amol Lokhande, C. Venkateswaran, M. Ramachandran, C. Vidhya, R. Kurinjimalar. " A Study on Various Implications on Reusing in Manufacturing", REST Journal on Emerging trends in Modelling and Manufacturing, 7(2), (2021): 63 -69.

39. Amol Lokhande, C. Venkates waran, M. Ramachandran, S. Chinnasami, T. Vennila."A Review on Various Implications on Re engineering in Manufacturing", REST Journal on Emerging trends in Modelling and Manufacturing, 7(3), 2021:70-75.

40. P. K. Chidambaram, Amol Lokhande, M. Ramachandran, Vimala Saravanan, Vidhya Prasanth, "A Review on Biodiesel Properties and Fatty acid composites", REST Journal on Emerging trends in Modelling and Manufacturing, 7(3), 2021:87-93.

41. P.K.Chidambaram, A mol Lokhande, M. Ramachandran, M. Nathiya, G. Mathivanan, " A study on Carbon Fiber Based Poly mer Rein Force composites", REST Journal on Emerging trends in Modelling and Manufacturing, 7(3), (2021): 94100.

42. Kurinjimalar Ramu, M. Ramachandran, M. Nathiya, M. Manjula "Green Supply Chain Management; with Dematel MCDM Analysis" Recent trends in Management and Commerce, 2(3), (2022): 8-15.

43. Amol Lokhande; C. Venkates waran, M. Ramachandran, C. Sathiyaraj, K. Nathiya, "Recycling Process Impact in Current Scenario Manufacturing A Study", Recent trends in Management and Commerce, 2(1), (2021):20-25.

44. Vimala Saravanan, M. Ramachandran, T. Vennila, G. Mathivanan "A Study on Multi-Objective Optimization on the basis of Ratio Analysis", Recent trends in Management and Commerce, 2(3), (2022):16-22

45. Soniya Sriram, M. Ramachandran, Sathiyaraj Chinnasamy, G. Mathivanan "A Review on Multi-Criteria DecisionMaking and Its Application", REST Journal on Emerging trends in Modelling and Manufacturing, 7(4), (2022):101-107.

46. S. Chinnasami, M. Ramachandran, P. Vidhya, M. Gowri "Study of Executive Director for Administrative Services on Moyamoya Disease and Energy application" REST Journal on Emerging trends in Modelling and Manufacturing, 7(4), (2022):116-124.

47. Sathiyaraj Chinnasamy, M. Ramachandran, Kurinjimalar Ramu, P. Anusuya "Study on Fuzzy ELECTRE Method with Various Methodologies" REST Journal on Emerging trends in Modelling and Manufacturing, 7(4), (2022):108-115.

48. Chinnasami Sivaji, M. Ramachandran, Kurinjimalar Ramu, Soniya Sriram, “A Review on Weight Process Method and its Classification” Data Analytics and Artificial Intelligence, 1(1), (2021): 1-8.

49. M. A mudha, M. Ramachandran, Vimala Saravanan, P. Anusuya, R. Gayathri, “A Study on TOPSIS MCDM Technies and its Application" Data Analytics and Artificial Intelligence, 1(1), (2021):9-14.

50. Vikrant Sharma, M. Ramachandran, Sathiyaraj Chinnasamy, Vimala Saravanan, "A Review on Structural Equation Modeling and Its Classification" REST Journal on Emerging Trends in Modelling and Manufacturing" 7(4), (2021):135-142.

51. Venkateswaran, Dr C. "Family Responsibilities Make a Barrier in the Career of Female Faculty." Mrs. Deepa Sharma, Dr. C. Ven kateswaran." Family Responsibilities Make a Barrier in the Career of Female Facu lty". International Journal of Computer Engineering In Research Trends (IJCERT), ISSN (2020): 2349-7084.

52. Sharma, Deepa, and DR C. VENKATESWARAN. "Discrimination Face Female Faculty During the Recruitment \& Selection and Train ing Time in The Academic Sector." Journal of Contemporary Issues in Business and Govern ment 27, no. 3 (2021): 1104-1108.

53. Kaur, Mandeep, and Dr C. Venkateswaran. "To Study the Work Life Balance among Working Women, Post Maternity in Banking Sector." International Journal of Management (IJM) 11, no. 2 (2020). 